•综述・

\title{
植物大年结实及其与动物㫟食行为之间的关系
}

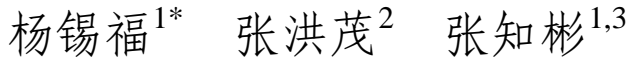 \\ 1 (中国科学院动物研究所农业虫害鼠害综合治理研究国家重点实验室, 北京 100101) \\ 2 (华中师范大学生命科学学院, 武汉 430079) \\ 3 (中国科学院生物互作卓越创新中心, 北京 100049)
}

\begin{abstract}
摘要: 大年结实(mast seeding)是多生年植物种群周期性同步大量繁殖的一种自然现象。大年结实作为植物适应环 境条件、提高繁殖能力的一种策略而备受关注, 但其驱动机制和进化意义尚存在较大争议。在依赖动物扩散种子 的植物中, 大年结实被认为是一种调控动物贴食行为、提高种子扩散效率, 并最终增加繁殖成功率的一种策略; 动 物介导的植物间互作可能是促进植物共存的进化驱动力。本文简要梳理了大年结实现象的各种假说, 提出了一个 包括气候、资源、动植物互作的理解大年结实机制的概念框架, 并着重讨论了大年结实和动物近食行为之间的关 系及其进化和生态意义。建议未来研究需要借助长期生态监测和分子生物学方法, 揭示植物大年结实与动物贴食 行为之间的生态与进化过程。
\end{abstract}

关键词: 大年结实; 种子扩散; 植物更新；捕食者饱和假说; 动物扩散假说; 分散这藏

\section{Mast seeding and its relationship to animal hoarding behaviour}

\author{
Xifu Yang ${ }^{1 *}$, Hongmao Zhang ${ }^{2}$, Zhibin Zhang ${ }^{1,3}$ \\ 1 State Key Laboratory of Integrated Management of Pest Insects and Rodents in Agriculture, Institute of Zoology, \\ Chinese Academy of Sciences, Beijing 100101 \\ 2 School of Life Sciences, Central China Normal University, Wuhan 430079 \\ 3 CAS Center for Excellence in Biotic Interactions, University of Chinese Academy of Sciences, Beijing 100049
}

\begin{abstract}
Mast seeding is a natural phenomenon that large seeding perennial plants perform periodic and synchronous reproduction. Mast seeding has been regarded as a strategy to adapt to environmental conditions and improve their reproductive ability, but its underlying mechanism and eco-evolutionary implications remain controversial. In animal dispersed reliant plants, mast seeding is considered as a scatter-hoarding protection strategy to improve dispersal efficiency and ultimately increase reproductive success. However, animal-mediated indirect seed-seed interactions of sympatric tree species may be an evolutionary driver of plant coexistence. Here, we briefly review the various hypotheses that have been suggested to explain the phenomenon of mast seeding, and we propose a conceptual framework integrating climate, resources and interaction between plants and animals to disentangle the mechanism of mast seeding. We also discuss the interaction between mast seeding and animal hoarding behavior and its evolutionary and ecological implications. In the future, long-term ecological monitoring and molecular biology methods should be used to reveal the ecological and evolutionary processes between mast seeding and animal hoarding behaviour.
\end{abstract}

Key words: mast seeding; seed dispersal; plant regeneration; predator satiation hypothesis; animal dispersal hypothesis; scatter hoarding

许多植物在某些年份同步大量繁殖, 而在其他 年份很少或根本不繁殖, 这一现象普遍存在于自然 界中。生物学家将这种多年生植物种群周期性地(如 间隔2-5年)同步大量繁殖的现象, 称为大年结实 (masting or mast seeding) (Silvertown, 1980; Kelly, 1994; Herrera et al, 1998)。大年结实包含3个关键要 素: 变异性(variability)、同步性(synchrony)、周期性 (periodicity) (Kelly \& Sork, 2002)。由于大年结实带

收稿日期: 2019-10-22; 接受日期: 2020-02-22

基金项目: 国家重点研发计划(2017YFC0503802)、国家自然科学基金(31330013)和中国博士后科学基金(2019M650840)

* 通讯作者 Author for correspondence. E-mail: yangxifu@ioz.ac.cn 
来了一系列明显的选择性劣势, 包括在种子小年减 少了繁殖机会(Waller, 1993)和在种子大年幼苗更严 重的密度制约性死亡(Hett, 1971), 例如一些竹类在 一次盛花或大量结实后随即死亡的极端现象 (Janzen, 1976; Keeley \& Bond, 1999), 因此阐明大 年结实的进化与生态意义, 不仅有助于理解物种共 存机制, 而且对评估物种多样性维持中随机过程和 更新生态位(regeneration niche)也具有重要的作用。 此外, 结合不同纬度的种子产量变化及其种间差异, 也可以解释物种多样性的纬度梯度格局(Wright et al, 2005; Usinowicz et al, 2017)。

植物更新是森林生态系统功能维持与健康运 转的关键。许多结实大种子的植物通常依赖动物(如 鼠类、鸟类)扩散种子, 也即在种子成熟季节, 䛎藏 种子的动物会将种子搬离母树埋藏在枯枝落叶或 土壤浅层, 其中一部分种子会逃脱动物捕食和真菌 侵蚀, 并遇到合适的环境条件而萌发生成幼苗, 帮 助植物实现了种子传播和更新。因此, 动物的种子 贮藏行为对植物更新有重要影响(Vander Wall, 2002; Pesendorfer et al, 2016b)。为了能更好地生存繁殖, 植物表现出大年结实, 这一现象可能是植物适应环 境尤其是生物环境(如种子捕食者)的结果: 因为植 物必须从大年结实中获益, 即植物在高繁殖努力下 提高繁殖效率(又称规模经济, economy of scale, EOS) (Norton \& Kelly, 1988; Wright et al, 2005; Żywiec et al, 2012), 以逃避动物的过度捕食和促进 动物分散败藏。动物根据植物种子产量而改变其咜 食行为策略, 以适应植物生产的变化。例如, 在美 国东部落叶森林, 大年结实引发鼠类、鹿和舞毒蛾 等种群波动(Ostfeld et al, 1996)。此外, 植物大年结 实常伴有大量的幼苗建成, 因此, 这种生殖特征影 响着植物种群的动态和分布格局, 进而可能影响到 动植物群落的结构组成, 甚至影响整个生态系统, 对阐明森林生态系统生物多样性、稳定性维持机制 具有重要作用(Kelly，1994; Kelly \& Sork，2002; Crone et al, 2009; Bogdziewicz et al, 2016)。

自20世纪70年代以来, 大年结实现象受到生物 学家和生态学家的广泛关注(Janzen, 1971; Kelly, 1994; Norghauer \& Newbery, 2011; Koenig et al, 2015), 并且相继提出了一些假说 (如: 资源匹配假
说、捕食者饱和假说、风媒授粉假说、物候同步假 说等)以阐明大年结实现象的原因和机制。然而学界 一直存在两种争论，即大年结实是气候因子造成的 结果, 还是一种进化繁殖策略(见: Kelly \& Sork, 2002)。为了解决这一争论, Kelly和Sork (2002)试图 从植物进化的角度综述之前的研究，重点从资源匹 配假说、风媒授粉假说和捕食者饱和假说等方面分 析了大年结实现象潜在的原因和驱动机制; 他们认 为大年结实是植物的一种适应性的繁殖策略，且受 气候因素的直接影响。Crone和Rapp (2014)重点从 资源预算假说(the resource budget hypothesis，即大 年结实所需要的资源比植物一年累积的还要多)对 大年结实的机制进行了概述。Pearse等(2016)提出了 一种通过开花诱导、授粉和果实成熟，将气候、资 源与大年结实联系起来的概念图, 并对大年结实的 机制进行了综述。国内，陈波等(2003)对早期植物生 殖生态学中大年结实的相关研究进展进行了综述; 杜彦君和马克平(2012)对种子产量的时空变化格局 进行了概述; 殷东生和沈海龙(2016)重点从资源分 配的角度就大年结实对营养生长和林木资源消耗 的影响及主要影响因素进行了综述。

然而，以上综述主要是从植物的角度来考虑， 更多关心的是植物自身的策略，而对大年结实与动 物行为策略之间关系的总结较少 (Vander Wall, 2010)。本文试图从植物大年结实现象的成因和驱动 机制、大年结实和动物咜食行为、大年结实的进化 和生态意义, 以及现有研究的局限和展望等方面加 以概述和分析，以期对未来深入研究大年结实的原 因和机制提供参考。

\section{大年结实的驱动机制}

植物为什么会间断性地生产大量的果实和种 子? 生物学家和生态学家们曾提出了不同的假说, 旨在揭示这一现象的潜在机制。本文梳理了各种解 释大年结实现象的假说(表1), 重点概述了与动物介 导的植物种子传播和更新有关的捕食者饱和假说、 动物扩散假说和大种子假说，简要分析了大年结实 现象的近因 (proximate causes) 和远因 (ultimate causes), 提出了一种综合理解大年结实现象的概 念框架。 
表1 解释大年结实现象的各种假说

Table 1 Hypotheses which have been proposed to explain the phenomenon of mast seeding

\begin{tabular}{|c|c|c|c|}
\hline $\begin{array}{l}\text { 假说 } \\
\text { Hypothesis }\end{array}$ & $\begin{array}{l}\text { 描述 } \\
\text { Description }\end{array}$ & $\begin{array}{l}\text { 典型特征 } \\
\text { Typical characteristics }\end{array}$ & $\begin{array}{l}\text { 评论 } \\
\text { Comment }\end{array}$ \\
\hline $\begin{array}{l}\text { 资源匹配(气候跟踪) } \\
\text { Resource matching } \\
\text { (Weather tracking) }\end{array}$ & $\begin{array}{l}\text { 种子产量的变化是由于植物可 } \\
\text { 利用资源的变化造成的 } \\
\text { Seed crops vary in response to } \\
\text { plant available resources vari- } \\
\text { ation (Norton \& Kelly, 1988) }\end{array}$ & $\begin{array}{l}\text { 在不改变营养生长或储备 } \\
\text { 资源的情况下实现种子产 } \\
\text { 量的变化 } \\
\text { Variable seed crops achiev- } \\
\text { ed without switching re- } \\
\text { sources from vegetative } \\
\text { growth or reserves }\end{array}$ & $\begin{array}{l}\text { 较早被提出，早期采用反证法; 多数支持而少数不完 } \\
\text { 全支持 } \\
\text { Put forward earlier, and early adopt proof by } \\
\text { contradiction (Kelly \& Sork, 2002); more evidence in } \\
\text { support (Kelly, 1994; Pearse et al, 2014; Monks et al, } \\
\text { 2016) and little in incomplete support (Koenig \& Knops, } \\
\text { 2000; Żywiec et al, 2012; Nussbaumer et al, 2018) }\end{array}$ \\
\hline $\begin{array}{l}\text { 捕食者饱和 } \\
\text { Predator satiation }\end{array}$ & $\begin{array}{l}\text { 大量结实使捕食者饱和，导致 } \\
\text { 个体被捕食的比例降低 } \\
\text { Large seed crops satiate seed } \\
\text { predators, which thus lead to in- } \\
\text { dividuals experience lower pro- } \\
\text { portion seed predation (Salis- } \\
\text { bury, 1942; Janzen, 1971) }\end{array}$ & $\begin{array}{l}\text { 种子大年捕食率较低 } \\
\text { Lower predation rate in } \\
\text { high-seed years }\end{array}$ & $\begin{array}{l}\text { 被多数研究支持; 不过, 取决于功能群的类型 } \\
\text { Well supported by many studies (Janzen, 1976; Sork, } \\
\text { 1983; Smith et al, 1990; Kon et al, 2005; Fletcher et al, } \\
\text { 2010; Xiao et al, 2013b; Wang et al, 2017; Bogdziewicz } \\
\text { et al, 2018); however, depends on the types of functional } \\
\text { groups. }\end{array}$ \\
\hline
\end{tabular}

风媒授粉

Wind pollination
风媒授粉植物同步大量开花可 以获得更高的传粉效率

Wind-pollinated plants can achieve greater pollination efficiency through synchronized above-average flowering effort (Nilsson \& Wastljung, 1987)

动物扩散(捕食者扩散) 大年结实吸引更多扩散者, 因

Animal dispersal (Predator dispersal) 而刺激分散陉藏动物更多的扩 散和陉藏种子

Large fruit production attract more seed dispersers, and result in wider dispersal and hoard by scater-hoarders (Janzen, 1971; Kelly, 1994)

环境预测 植物可以“预知”最适气候条件

Environmental prediction 而繁殖和建成

Plants can "predict" favorable climate conditions for reproduction and establishment (Smith et al, 1990; Kelly, 1994)

\begin{tabular}{|c|c|}
\hline $\begin{array}{l}\text { 大种子 } \\
\text { Large seed }\end{array}$ & $\begin{array}{l}\text { 生产大种子增加了种子大年间 } \\
\text { 的恢复时间 } \\
\text { Selection for larger seed size } \\
\text { increases the recovery time be- } \\
\text { tween large seed crops (Sork, } \\
\text { 1993) }\end{array}$ \\
\hline $\begin{array}{l}\text { 动物传粉 } \\
\text { Animal pollination }\end{array}$ & $\begin{array}{l}\text { 大量开花更易于吸引动物传粉 } \\
\text { 者, 从而提高传粉效率 } \\
\text { Large flowering attract animal } \\
\text { pollinators, thus enhance polli- } \\
\text { nation efficiency (Norton \& } \\
\text { Kelly, 1988; Sun et al, 2007) }\end{array}$ \\
\hline $\begin{array}{l}\text { 辅助成本 } \\
\text { Accessory cost }\end{array}$ & $\begin{array}{l}\text { 较高的固定繁殖成本有利于更 } \\
\text { 少、更大规模的繁殖活动 } \\
\text { Higher fixed reproductive costs } \\
\text { favor fewer, larger reproductive } \\
\text { episodes (Lalonde \& Roitberg, } \\
\text { 1992; Kelly, 1994) }\end{array}$ \\
\hline $\begin{array}{l}\text { 捕食者净化 } \\
\text { Predator cleansing }\end{array}$ & $\begin{array}{l}\text { 竹子的同步性死亡降低了以成 } \\
\text { 年树叶为食的植食性动物的密度 } \\
\text { The synchronized death of bam- } \\
\text { boos reduces densities of herbi- } \\
\text { vores feeding on adult leaves } \\
\text { (Pearson et al, 1994) }\end{array}$ \\
\hline
\end{tabular}

在大量开花年受精比例更 高

Higher \% fertilization in

high flowering years

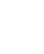

很好地被验证; 然而, 部分实验结果是模棱两可的 Well documented (Norton \& Kelly, 1988; Smith et al, 1990; Kelly, 1994; Kon et al, 2005; Crone et al, 2009; Moreira et al, 2014); however, some test results are ambiguous (Kelly et al, 2001)

种子大年扩散率更高或扩 散更远

Higher seed dispersal rate or greater dispersal distances in high-seed years

种子或幼苗在大年结实时 生存率更高

Higher seed or seedling survival in mast seeding

在种子较大的物种中, 种 子数随年际变化更大 More variable seed number among years in related species with large seeds

在大量开花年受精比例更 高 Higher \% fertilization in high-flowering years

是否有利于持续繁殖取决于扩散者的功能反应; 部分 结论支持, 部分反对

Whether favor constant reproduction, depending on functional response of dispersers; some evidences in support (Silvertown, 1980; Vander Wall, 2002; Vander Wall et al, 2005; Li \& Zhang, 2007; Pesendorfer \& Koenig, 2016; Pesendorfer et al, 2016b; Zwolak et al, 2016) or against (Koenig et al, 1994; Xiao et al, 2013b)

部分证据支持, 部分反对

Some evidences in support (Williamson \& Ickes, 2002;

Piovesan \& Adams, 2005; Burns, 2012) or against (Koenig et al, 2010)

部分结论支持, 部分反对; 其他研究认为种子大小和种 子数量存在权衡; 中等大小的种子具有更高的建苗率 Some support (Jansen et al, 2004) or against (Kelly, 1994); other studies have suggested a trade-off between seed size and number (Xiao et al, 2015); medium-sized seeds have a higher rate of seedling establishment (Cao et al, 2016)

一些分布稀少的植物个体同时产生大量的花, 更有助 于种群数量的增加

A few sparsely distributed individuals that simultaneously produce a large number of flowers are more conducive to population growth (Norton \& Kelly, 1988)

在植物内进行大量繁殖时，不需要植物个体之间的同步生产，因此不能单独解释 每粒种子的辅助成本较低 大年结实; 可能夸大了大小年间的变化

Lower accessory costs per seed for large reproductive efforts within a plant

Does not require synchrony between individuals, hence cannot alone explain mast seeding; may exaggerate variation between high and low years (Kelly, 1994)

\section{群体开花可能是为了逃避 较少验证}

捕食 Less empirical test

Gregarious flowering may escape predation 
表1 (续) Table 1 (continued)

\begin{tabular}{|c|c|c|c|}
\hline $\begin{array}{l}\text { 假说 } \\
\text { Hypothesis }\end{array}$ & $\begin{array}{l}\text { 描述 } \\
\text { Description }\end{array}$ & $\begin{array}{l}\text { 典型特征 } \\
\text { Typical characteristics }\end{array}$ & $\begin{array}{l}\text { 评论 } \\
\text { Comment }\end{array}$ \\
\hline $\begin{array}{l}\text { 大年抑制 } \\
\text { Mast depression }\end{array}$ & $\begin{array}{l}\text { 植物种子产量的周期性变化导致 } \\
\text { 植食性动物种群的周期性波动 } \\
\text { The cyclic population fluctuation } \\
\text { of herbivores is caused by the } \\
\text { cyclic seed crops of plants } \\
\text { (Selås, 1997) }\end{array}$ & $\begin{array}{l}\text { 高种子产量的生产是以牺牲对 } \\
\text { 植食性动物的化学防御为代价 } \\
\text { 的 } \\
\text { The production of high-seed cro- } \\
\text { ps is at the cost of chemical de- } \\
\text { fense against herbivores }\end{array}$ & $\begin{array}{l}\text { 主要用种子内化学防御物质的多少来解释植物 } \\
\text { 与捕食者间的关系 } \\
\text { The relationship between plants and predators is } \\
\text { largely explained by the amount of chemical de- } \\
\text { fenses in seeds }\end{array}$ \\
\hline $\begin{array}{l}\text { 异型杂交 } \\
\text { Outcrossing }\end{array}$ & $\begin{array}{l}\text { 大年结实有利于杂交繁殖 } \\
\text { Mast seeding facilitates out- } \\
\text { breeding (Tisch \& Kelly, 1998) }\end{array}$ & $\begin{array}{l}\text { 大量开花可减少近交衰退 } \\
\text { Mast seeding may reduce inbre- } \\
\text { eding depression }\end{array}$ & $\begin{array}{l}\text { 没有得到很好验证 } \\
\text { Not well supported }\end{array}$ \\
\hline $\begin{array}{l}\text { 竹子火烧循环 } \\
\text { Bamboo fire cycle }\end{array}$ & $\begin{array}{l}\text { 竹子等无性系种群大年盛花后 } \\
\text { 同步死亡, 这助长了火灾的发 } \\
\text { 生, 从而阻止了树木与竹子之 } \\
\text { 间的竞争 } \\
\text { Synchronized death of clonal } \\
\text { populations such as bamboos } \\
\text { after high flowering encourages } \\
\text { fire, which prevents trees from } \\
\text { out-competing the bamboo } \\
\text { (Keeley \& Bond, 1999) }\end{array}$ & $\begin{array}{l}\text { 竹类等植物依赖林窗更新和扩 } \\
\text { 散种群的一种适应对策 } \\
\text { An adaptive strategy for plants } \\
\text { such as bamboo that depends on } \\
\text { forest gap regeneration and di- } \\
\text { spersal }\end{array}$ & $\begin{array}{l}\text { 仅是一种推论, 需要实验验证 } \\
\text { It is just a inference that needs to be tested experi- } \\
\text { mentally }\end{array}$ \\
\hline $\begin{array}{l}\text { 物候同步 } \\
\text { Phenological synchrony }\end{array}$ & $\begin{array}{l}\text { 大年结实是由个体的资源限制 } \\
\text { 和环境变化引起的种群范围的 } \\
\text { 花粉可利用性共同导致 } \\
\text { The mast seeding is caused by } \\
\text { both individual resources limi- } \\
\text { tation and population-wide poll- } \\
\text { en availability due to environ- } \\
\text { mental variation (Koenig et al, } \\
\text { 2015; Pesendorfer et al, 2016a) }\end{array}$ & $\begin{array}{l}\text { 物候的作用导致植物同步开花 } \\
\text { 从而增加授粉的有效性 } \\
\text { Phenology leads to synchronous } \\
\text { flowering of plants, increasing } \\
\text { pollination effectiveness }\end{array}$ & $\begin{array}{l}\text { 仅在山谷橡树中得到验证, 需更多实验验证 } \\
\text { Only documented in the valley oak (Koenig et al, } \\
\text { 2015; Pesendorfer et al, 2016a), more experiments } \\
\text { are needed }\end{array}$ \\
\hline
\end{tabular}

\section{1 捕食者饱和假说 (the predator satiation hypothesis)}

Salisbury (1942)发现山毛榉(Fagus crenata)和 橡树的种子在结实大年不会被昆虫全部取食, 据此 提出植物周期性大量结实可能是为了避免种子被 过度捕食的观点(Salisbury, 1942)。此后, Janzen $(1971,1976)$ 在研究竹类时发现, 捕食者饱和是使竹 类物种大年结实的原因之一。这一发现也引发了生 物学家对植物大年结实的广泛关注。此后, 捕食者 饱和假说被提出, 该假说认为植物大年结实可以使 种子捕食者(如昆虫、脊椎动物)的捕食效应饱和，从 而增加种子存活的机会, 提高繁殖适合度(Janzen, 1971)。随后大量的研究证实大年结实时种子被捕食 率较低(Smith et al, 1990; Sork, 1993; Kon et al, 2005; Bogdziewicz et al, 2018)。近年来, 在依靠动物扩散 种子的植物中, 捕食者饱和效应得到了较好的验证 (Fletcher et al, 2010; Xiao et al, 2013b)。例如, Xiao 等 (2013b) 在四川都江堰地区对油茶 (Camellia oleifera) 种子产量、鼠类数量和种子命运等长达 8 年 的研究结果表明, 种子结实大年, 种子被鼠类搬运
和贮藏率更低、有更多种子存留和幼苗生成，支持 捕食者饱和假说(Xiao et al, 2015; Wang et al, 2017)。 虽然捕食者饱和假说被普遍认为可以有效解释大 年结实现象(Silvertown, 1980; Sork, 1993; Kelly \& Sork, 2002), 但仍有不足。例如, 支持捕食者饱和假 说的多数证据来自专食性(specialist)的昆虫捕食者 (Sork, 1993; Bogdziewicz et al, 2018), 较少来自泛 食性(generalist)的脊椎动物捕食者(如鼠类) (Vander Wall, 2010)。

\section{2 动物或捕食者扩散假说 (the animal or predator dispersal hypothesis)}

该假说认为大年结实通过提高种子在扩散后 逃脱捕食的机会, 促进了以动物(如分散捾藏鼠类 和鸟类)为媒介的植物的种子扩散(Janzen，1971; Silvertown, 1980; Vander Wall, 2002)。早期Kelly (1994)将该假说定义为: 大量果实更易吸引泛食性 的动物扩散者, 或者诱发分散陉藏者广泛扩散。这 个假说包含两个关键的预测: 在种子大年，种子扩 散速率更快、扩散距离更远。一般来说，鼠类和鸦 科鸟类面对大量种子时，会吃掉部分种子而扩散咜 
藏剩余的种子, 是大种子植物的重要扩散者(Vander Wall et al, 2005; Pesendorfer et al, 2016b); 而有些食 种子动物却是纯粹的种子捕食者, 因此大年结实可 能对依赖食种子动物扩散的植物不利(Vander Wall, 2010)。Vander Wall (2002)通过模拟大年结实, 结果 表明动物介导的种子扩散可能是一种选择力, 伴随 着捕食者饱和与高效的风媒授粉, 因而导致一些松 树种子产量在时空上发生变化。由于一个种子扩散 者的潜在效率不仅是对种子数量的反应, 而且取决 于扩散者对大年结实的功能反应，而不同类型的种 子扩散者可能对大年结实的功能反应不同，因而难 以完美地验证动物扩散假说(Koenig et al, 1994; Jansen et al, 2004; Xiao et al, 2013b)。近来的几项研 究分别通过调查鸦科鸟类如圣岛丛鸦(Aphelocoma insularis)和西从鸦 (A. californica) 对壳斗科植物种 子命运的影响 (Pesendorfer \& Koenig, 2016; Pesendorfer et al, 2016b), 以及黄喉姬鼠(Apodemus flavicollis)对欧洲山毛榉(Fagus sylvatica)种子的命 运的影响(Zwolak et al, 2016), 很好地支持了动物扩 散假说。

\section{3 大种子假说(the large seed hypothesis)}

该假说认为, 一些植物结实大种子年和小种子 年交替出现, 大种子有利于种子扩散和幼苗生成, 而且一些柇树的种子会随着大年结实时间间隔的 延长而增大(Sork, 1993)。植物倾向于结实大种子的 进化动力会增加大年结实的间歇时间, 因为结实大 种子需要更多时间积累资源(Sork, 1993), 具有大小 种子结实交替习性的植物在结实小种子年积累资 源是为了结实大种子。Jansen等(2004)在3年内追踪 了616粒首次被鼠类分散败藏的苦油楝(Carapa procera) 种子的命运, 发现大种子和高产量会刺激 鼠类更多地扮演种子扩散者而不是捕食者, 因此, 分散咜藏的鼠类倾向于选择高产量和大种子，这将 促使植物大量结实。但是, Kelly (1994)认为该假说 不能解释大年结实现象。Xiao等(2015)提出了一个 基于种子大小和产量的种子存活与扩散权衡框架, 表明种子存活与扩散权衡在种子水平和树个体或 种群水平的表现不同: 在种子水平上表现为大种子 有利于扩散, 而小种子有利于存活; 在个体或种群 水平上表现为种子产量低有利于扩散, 种子产量高 有利于存活。Cao等(2016)发现中等大小的假海桐 (Pittosporopsis kerrii)种子具有更高的建苗率。

\section{4 大年结实的近因和远因}

在阐明大年结实机制的研究中，常涉及到近因 和远因的探讨。近因是指影响植物在某一年份是否 能大量结实的直接和/或间接因素, 而远因则是对 植物繁殖进化对策的解释(Kelly, 1994)。研究表明, 有3组近因被认为是重要的: (1)环境因素(Schauber et al, 2002; Perez-Ramos et al, 2015; Bogdziewicz et al, 2020)。例如, Perez-Ramos等(2015)对地中海橡树 产量与环境关系的研究表明, 气温升高可能对常绿 植物种子产量产生不利影响; 在干旱条件下，落叶 物种的种子产量下降幅度可能会更大。然而, Wright 等(1999)在热带雨林中研究厄尔尼诺-南方涛动(El Niño-Southern Oscillation, ENSO)对植物种子产量 的影响, 表明厄尔尼诺-南方涛动带来的温暖、干燥 和阳光充足的条件会促进种子生产 (Detto et al, 2018); Kelly等(2013)研究表明，年际间温度升高有 利于种子大年。(2)传粉有效性与受精成功(Koenig \& Knops, 2005; Koenig et al, 2012)。例如, Koenig等 (2012)研究表明，花粉限制严重影响了橡树种子产 量, 甚至可能影响其他关键的生活史特征。(3)资源 分配的时间模式和生殖的生理成本(Crone et al, 2009; Sala et al, 2012)。例如, Crone等(2009)通过一 系列的实验，表明碳水化合物可控制单株植物的隔 年开花; 结实率会耗尽碳水化合物，从而阻止来年 开花; 当植物不同步开花时, 它们会减少生产种子 的数量, 从而防止碳水化合物过度消耗。以上这些 因素都是非排他性的，很可能共同起作用。例如， 环境因素可能通过影响每年可供繁殖的资源数量 而影响第二年的繁殖成功，进而影响传粉的有效性 (Nussbaumer et al, 2018)。

另一方面，风媒授粉、捕食者饱和与环境预测 等假说可能是造成植物大年结实的远因(如: Kelly, 1994)。Sork (1993)对温带和热带橡树大年结实现象 进行研究, 提出一种模型比较了 3 种不同假说以解 释橡树的进化意义, 主要考虑动物与植物互作的因 素，但没有考虑气候、资源限制的因素。Koenig等 (1994)对几种栋树的大年结实现象进行研究, 认为 这种现象是由风媒授粉和捕食者饱和共同决定; 风 媒授粉的有效性和气候条件的变化也是影响风媒 树种大年结实的远因(Houle，2001)。Kelly和Sork (2002)认为, 应从全球视角来研究植物的大年结实 
现象，不仅考虑气候和自然选择的影响，同时还应 考虑纬度、授粉和传播模式的影响 (Koenig \& Knops, 2000)。Pearse等(2016)对大年结实的机制进 行了综述, 并提出了一个概念图, 主要考虑气候和 资源限制的因素, 但没有考虑动物与植物互作; 他 们认为在理论上, 大年结实可以几乎完全由资源的 可利用性来驱动, 或完全由与植物资源无关的环境 因素来驱动, 而实际上, 资源和环境因素都是必要 的, 因此需共同考虑。最近, Bogdziewicz等(2020) 提出, 阐明大年结实的直接动力机制可从资源动 态、授粉限制、遗传和激素调节等3个主要过程来 讨论, 而环境变化虽然也是大年结实的一个驱动因 素, 但它在很大程度上是通过这些过程产生影响的, 因此, 环境变化作为一种大年结实驱动力的讨论可 纳入上述 3 个过程中。

我们的研究发现, 同域树种的同步或异步结实 会影响动物介导的植物种子之间的间接相互作用 (似然竞争、互惠或捕食); 这一选择压力反过来可 能促使大量植物向大年结实演化(Yang et al, 2020)。 因此, 这种物种之间的间接相互作用所导致的进化 过程值得深入研究。在Sork(1993)和Pearse等(2016) 提出的概念模型基础上, 本文提出一个包括气候、 资源、动植物互作的理解大年结实机制的概念框架 图(图1)。该框架图既考虑生态过程(实线部分), 也 考虑了进化过程(虚线部分)。生态作用包括: 大范 围适宜的气候提供了外部条件, 可导致植物同步开 花, 同步开花可能有利于吸引传粉者(如昆虫、鸟类) 异花授粉而提高传粉效率, 进而导致植物大年结 实。植物大年结实一方面通过饱和捕食者或刺激扩 散者而改变动物梡食行为, 进而促进动物分散咜藏 种子。种子被埋藏在适宜生境, 部分被动物遗忘的 种子从而有机会萌发、建成幼苗。植物大年结实另 一方面会大量消耗植物储备的资源, 导致次年植物 生长和繁殖资源匮乏, 形成负反馈调节, 进而抑制 植物同步开花。植物通过调控结实的时间间隔，从而 形成植物大小年之分。

进化选择作用包括：植物在进化过程中，为了 适应环境以使繁殖适合度最大化, 通过遗传变异和 分泌激素促使周期性大量同步开花进而吸引大量 传粉者以提高传粉效率, 最终导致植物周期性同步 大量结实。此过程不仅有利于植物资源的周转, 而

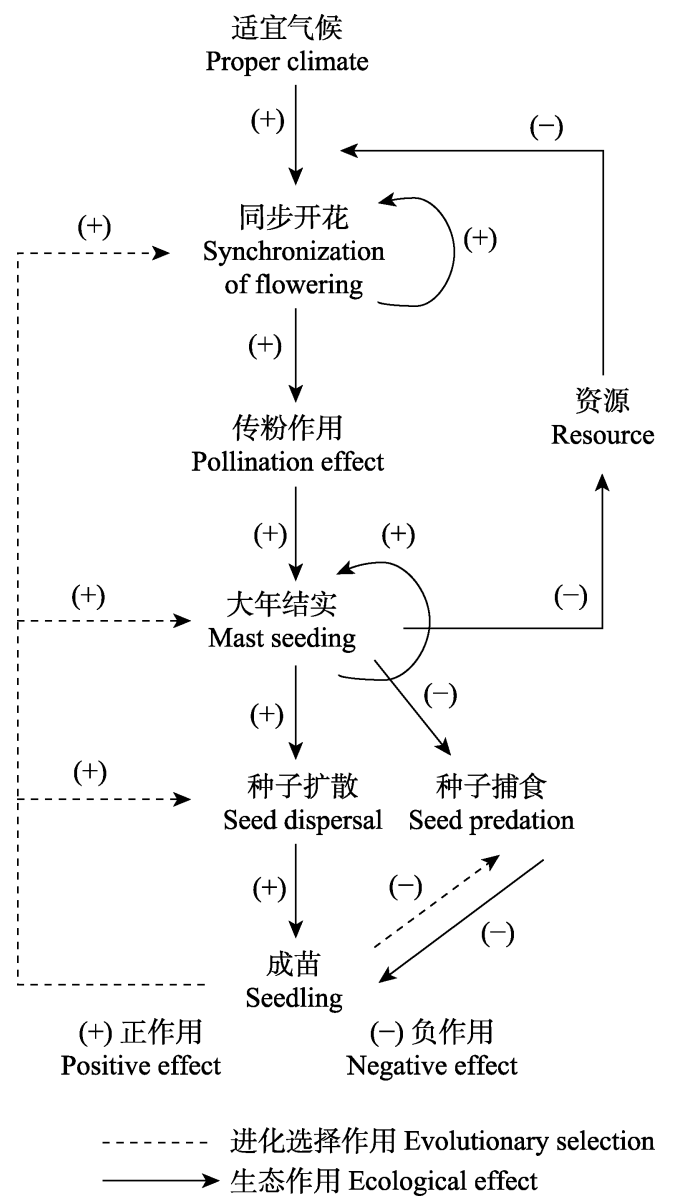

图1 一个整合气候、资源和动植物互作的进化与生态过程 的大年结实概念框架图

Fig. 1 A frame-diagram explaining the evolutionary and ecological processes of mast seeding by integrating the climate and resource and plant-animal interactions

且有利于调控动物种群数量及改变动物咜食行为, 促进动物对种子的扩散，避免动物对种子的过度捕 食，从而使动植物协同进化并处于一种动态平衡状 态。这种动态平衡对于维持森林生态系统的生物多 样性、稳定性及健康至关重要。

\section{2 大年结实与动物贴食行为}

植物间断性地大量结实是多因素综合作用的 结果, 而以植物种子(果实)为食的动物也必然会受 到植物种子产量波动的影响, 因此动物也会采用相 应的适应性策略(Vander Wall, 2010; Selås, 2016)。例 如, Granados等(2019)研究发现，通过砍伐改变植物 生物量, 在食物资源较充足的年份增加了脊椎动物 的迁移和繁殖。大年结实与动物行为之间的相互关 系是一个广泛的话题, 动物行为涉及咜食、领域、 
繁殖和社会行为等等。动物咜食行为对植物的自然 更新有着非常重要的作用, 植物的自然更新包括种 子生产、种子扩散、幼苗建成等过程, 其中, 种子 扩散是决定植物更新和扩散的关键阶段(Nathan \& Muller-Landau, 2000); 动物的咜食行为受植物大年 结实影响(Vander Wall, 2010)。限于篇幅, 本节重点 概述大年结实与动物它食行为之间的相互关系。

\section{1 动物陉藏行为}

动物的咜藏行为可分为集中败藏(larder hoarding)和分散咜藏 (scatter hoarding) (Vander Wall, 1990)。集中穴藏是指动物将收集的植物种子集中咜 藏在其洞穴或临时栖居场所, 通常败藏点少, 每个 贮藏位点种子数量多; 分散䛎藏是指动物将收集的 种子捾藏在多个聍藏点, 每个陉藏点的种子数量少, 通常仅有 1 粒种子。分散贮藏较集中陉藏更有利于 植物种子传播和更新, 因为动物往往将种子埋藏在 枯枝落叶或土壤浅层等适宜萌发和建成幼苗的微 生境, 这些种子一旦逃脱动物捕食和真菌侵蚀, 就 有机会萌发、建成幼苗, 实现植物更新。

种子产量的多少直接影响动物在越冬和繁殖 时潜在的食物供应(Vander Wall, 1990)。通过实验室 控制性实验如限食, 研究咜食动物在不同资源量条 件下(模拟种子产量大小年)的咜藏行为不失为一种 很好的手段。例如, 早期研究表明, 西伯利亚仓鼠 (Phodopus sungorus)在经历限食之后, 增加了对食 物的陉藏, 并且这种增加并不受限食时间长短的影 响(Day et al, 1999)。但是, 也有一些研究表明动物 在限食后的諚藏行为并没有受到显著影响(Wong \& Jones, 1985), 甚至减少了对食物的穴藏(Demas \& Bartness, 1999)。这可能与室内条件局限影响动物情 绪、行为等有关。

随着研究的不断深入、技术手段的提升, 半自 然围栏、野外实验证据也越来越多(Jansen et al, 2004; Fletcher et al, 2010; Xiao et al, 2013a, 2015; Zwolak et al, 2016)。例如, Jansen等(2004)在法属圭 亚那采用远程视频监控和线标法连续 5 年(3年种子 大年, 2 年种子小年)追踪了 3,000 粒苦油楝种子的命 运, 结果表明种子大年促进了鼠类䛎藏种子, 提高 了种子扩散成功率。对鸦科鸟类贮藏行为的研究也 表明，种子大年增加了鸟类对种子的栬藏 (Pesendorfer \& Koenig, 2016; Pesendorfer et al, 2016b)。

\section{2 种子扩散速率}

Vander Wall (2002)在内华达州西部的研究发现, 在不同年间鼠类对人工释放的杰弗里松 (Pinus jeffreyi)和糖松(P. lambertiana) 种子的扩散速率差异 显著, 种子大年鼠类对种子的扩散速度明显比种子 小年要快得多。然而, 也有很多研究与Vander Wall (2002)的结果相反。例如, Sork (1983)的研究表明, 在种子大年野外种子的消失速率比较缓慢, 动物仅 搬走很少一部分种子。Montiel和Montaña (2000)通 过对墨西哥奇瓦瓦沙漠南部的脊椎动物扩散胭脂 仙人掌属(Opuntia)植物果实的研究, 发现果实的消 失速率与果实的可获得性成反比: 在低密度种群中 和干旱的年份果实的消失速率是最快的。一些研究 也证实，种子在结实小年比结实大年被鼠类扩散得 更快(Li \& Zhang, 2007; Fletcher et al, 2010; Cao et al, 2017; Xiao \& Huang, 2019)。此外, 还有研究表明, 在种子大小年间油茶种子被鼠类扩散的速度没有 显著差异, 但却发现在油茶种群较大的斑块释放的 油茶种子被鼠类扩散得更快, 因此他们认为油茶种 子的绝对数量更能影响种子被扩散的速率(Xiao et al, 2005)。

\section{3 动物分散赂藏}

动物通过分散咜藏食物资源，不仅有利于自身 的生存和繁殖成功, 而且也有利于植物种子在合适 的生境萌发、幼苗建成和植物的更新(Vander Wall, 1990, 2010; Cao et al, 2017; Pesendorfer \& Koenig, 2017)。Li和Zhang (2007)研究发现, 种子大年增加 了鼠类分散咜藏山杏(Prunus armeniaca)种子的数 量; 而且在种子大年，山杏种子被分散咜藏的数量 是种子小年的4倍。Zhang等 (2008)在实验围栏条件 下增加油茶种子数量时发现, 小泡巨鼠 (Leopoldamys edwardsi)分散聍藏了更多的种子，支 持提出的大年结实促进咆藏假说 (the mastingenhanced hoarding hypothesis)。Xiao等(2013a)研究 发现, 与种子小年相比, 在种子大年岩松鼠 (Sciurotamias davidianus)虽然收获较少的橡子, 但 贮藏了更多收获的种子。Pesendorfer等(2016b)研究 发现, 种子大年圣岛丛鸦分散咜藏了更多种子。此 外, Vander Wall (2002)研究表明, 鼠类在种子大年 对埋藏种子的二次埋藏强度低于在种子小年。

然而, Jansen等(2004)认为植物大年结实在饱和 捕食者的同时，也会降低扩散动物分散栬藏种子的 
比例。Cao等(2017)研究表明, 较大的种子数量减少 了鼠类对假海桐种子的栬藏, 但较大的种子数量对 鼠类陉藏落瓣山茶(Camellia kissi)种子没有显著影 响。其他很多研究也表明, 鼠类分散拒藏植物种子 的比例在种子大小年间没有显著的差异(Theimer, 2001; Vander Wall, 2002; Xiao et al, 2005)。

\section{4 种子扩散距离}

一些研究认为, 在种子大年时, 鼠类有更多的 资源能够补充能量的消耗, 为降低种子被其他动物 盗食的概率，鼠类会将种子扩散更远(Vander Wall, 2002; Li \& Zhang, 2007; Vander Wall \& Beck, 2012)。 这些研究支持动物扩散假说。然而, 另一些研究认 为, 在植物种子大年时, 丰富的食物资源降低了咜 藏种子被盗食的概率, 因此鼠类也会降低对这些种 子的管理, 导致种子扩散距离较近(Moore et al, 2007)。很多研究证实在种子结实大年, 动物对种子 的扩散距离显著小于种子结实小年(Theimer, 2001; Jansen et al, 2004; Xiao et al, 2005; Cao et al, 2017; Xiao \& Huang, 2019)。例如, Cao等(2017)通过群落 水平的研究证实, 分散咜藏鼠类对假海桐和落瓣山 茶种子的扩散距离在种子大年均极显著小于种子 小年。Zwolak等(2016)研究发现, 欧洲山毛榉被鼠 类扩散的平均距离在种子小年是种子大年的近 8 倍。以上这两种截然相反的结果可能是因为, 动物 将植物种子扩散到远离母树的地方贮藏起来, 虽然 可以降低种子被其他动物盗食的概率, 但扩散种子 需要消耗大量的能量, 因此动物对种子的扩散距离 取决于种子资源量、动物扩散种子时的能量消耗, 以及败藏种子被盗食的概率之间的权衡(Moore et al, 2007; Solbreck \& Knape, 2017)。

\section{5 幼苗建成率}

植物大年结实可以使捕食者的食物饱和, 从而 使更多种子逃脱捕食而存活, 增加幼苗建成率 (Kelly, 1994; Kelly \& Sork, 2002)。研究证实, 一些 种子的幼苗建成率在种子大年时显著高于小年 (Vander Wall, 2002; Jansen et al, 2004)。例如, Vander Wall (2002) 发现在种子大年时, 西黄松 (Pinus ponderosa)、糖松和约弗里松种子的幼苗建成率分 别为种子小年的 2.2 倍、 4.1 倍和 6.9 倍; Jansen等 (2004)发现苦油楝种子的幼苗建成率在种子大年时 为小年的4.5倍。多数研究均发现在种子生产大年时 种子幼苗建成率比较高, 表明植物大年结实能够在
一定程度上促进植物幼苗的更新 (Kelly, 1994; Vander Wall, 2002; Joubert et al, 2013)。Zwolak等 (2016)连续4年 $(2+2)$ 追踪了 1,600粒欧洲山毛榉种 子的命运, 结果表明种子大年减少了黄喉姬鼠对埋 藏种子的找回，增加了幼苗出苗率。

\section{3 大年结实的进化和生态意义}

大年结实在植物更新、动植物互作以及许多其 他生态过程中起着至关重要的作用(Vacchiano et al, 2018; Bogdziewicz et al, 2020)。大年结实意味着食 物资源丰富, 因此有更多种子能够逃脱捕食者的捕 食(捕食者饱和假说) (Vander Wall, 1990; Jansen et al, 2004; Xiao et al, 2013b; Cao et al, 2017), 同时也会 刺激动物的号食行为, 览食动物在种子大年比在种 子小年贮藏更多种子(动物扩散假说) (Vander Wall, 2002; Li \& Zhang, 2007; Xiao et al, 2013a; Zwolak et al, 2016), 动物这种行为可能是对大年结实的进化 适应(Zhang et al, 2008); 这些被贮藏的种子将更有 机会建成幼苗, 从而促进植物种子扩散和种群更新 (Kelly \& Sork, 2002; Fletcher et al, 2010; Joubert et al, 2013)。此外, 同域树种的结实同步与否会改变动物 (如鼠类)介导的植物种子间的间接关系(Norghauer \& Newbery, 2011; Yang et al, 2020), 进而可能影响 植物物种之间的共存 (Chen \& Hsu, 2011; Garzon-Lopez et al, 2015)。通过理解像大年结实这 类复杂现象是如何发生的, 有利于更好地推断形成 这种现象的进化过程(West-Eberhardt, 2003)。

总之, 研究大年结实的机制和规律, 不仅具有 重要的理论意义, 而且有重大的实践意义。对动物 来说, 植物周期性地同步结实有助于维持动物多样 性(Silvertown, 1980; Kelly, 1994; Bogdziewicz et al, 2016; Granados et al, 2019); 对植物来说, 大年结实 提高了授粉效率, 使捕食者饱和, 大量种子被动物 搬离母树而分散咜藏在浅层土壤中, 不仅更易逃脱 捕食者的捕食, 且有利于种子萌发、幼苗建成, 直 至完成种群更新(Kelly et al, 2001; Kelly \& Sork, 2002; 孙明洋等, 2011; Pearse et al, 2016)。植物周期 性大量繁殖以适应环境变化, 动物群落对这些变化 快速响应, 又反过来影响植物空间格局、演替和恢 复过程, 从而形成协同进化。此外, 通过阐明大年 结实机制, 可以了解其如何以及为何可能对气候变 化和宏量营养素循环(macronutrient cycles)作出反 
应, 以帮助预测植被动态和生物地球化学循环 (Vacchiano et al, 2018; Bogdziewicz et al, 2020)。在 农业系统中, 对大年结实机制的认识有助于预测具 有商业价值的水果和坚果作物如苹果、柑橘和开心 果的种植时间(Smith \& Samach, 2013; 殷东生和沈 海龙, 2016)。在野生动物管理和人类健康方面, 更 好地理解与大年结实相关的资源脉冲 (resource pulses)时间, 有助于野生动物管理者掌握动物种群 的变化规律和公众预防莱姆病(Lyme disease)等潜 在的健康风险(Ostfeld et al, 2006)。

\section{4 展望}

尽管以往的研究提出的捕食者饱和、捕食者扩 散和大种子等假说为理解植物大年结实及其与动 物(特别是鼠类)咜食行为之间的关系提供了重要的 理论, 但将大年结实作为一种进化特征的很多关键 方面还有待阐明。近来的研究多倾向于考虑多种因 素的作用, 如Bogdziewicz等(2020)认为, 要全面剖 析大年结实现象的直接动力机制, 需要重点关注3 个主要过程: 资源动态、授粉限制、遗传和激素调 节。由于相对于多年生植物较长的生活史, 一般研 究的时间尺度均较短(多在10年左右或更短), 显然 不足以揭示多年生植物的生长和繁殖变化机制。未 来的研究需要通过长期生态监测对植物物种的结 实大小年周期进行精确确定, 并在此过程中调查生 态系统中动植物群落的变化规律, 以揭示植物大年 结实与包括动物它食行为在内的各种生态过程之 间的关系。

建议未来应关注以下 4 个方面的研究: (1)在个 体和种群水平上, 着重考虑规模经济和资源预算假 说, 加强对植物的长期观察研究和改变资源的控制 实验; 此外, 从微观水平研究植物遗传和激素对同 步开花、大年结实的调控机制, 利用基因组等分子 生物学方法深入挖掘植物大年结实的进化机制。(2) 在群落水平上, 探讨为什么有些植物物种的种子产 量是相对恒定的, 而同样环境下其他物种却出现大 年结实现象? 从动物(如鼠类、鸟类)介导的植物种 子间的间接相互作用(即动植物互作层面)来探讨大 年结实机制, 或许是一种新的研究思路。(3)在景观 或全球尺度上，通过长时间、多种因素共同考虑， 探讨哪些假说是互相排斥的或共同作用的, 哪些因 素是占主导作用, 哪些因素是影响大年结实的近因
或远因。(4)由于森林生态系统受到了人为干扰和全 球气候变化影响, 因此研究在人为干扰和全球气候 变化下植物的生产和繁殖策略也是未来研究应该 加强的方面。

\section{参考文献}

Bogdziewicz M, Ascoli D, Hacket-Pain A, Koenig WD, Pearse I, Pesendorfer M, Satake A, Thomas P, Vacchiano G, Wholgemuth T, Tanentzap A (2020) From theory to experiments for testing the proximate mechanisms of mast seeding: An agenda for an experimental ecology. Ecology Letters, 23, 210-220.

Bogdziewicz M, Espelta JM, Munoz A, Aparicio JM, Bonal R (2018) Effectiveness of predator satiation in masting oaks is negatively affected by conspecific density. Oecologia, 186, 983-993.

Bogdziewicz M, Zwolak R, Crone EE (2016) How do vertebrates respond to mast seeding? Oikos, 125, 300-307.

Burns KC (2012) Masting in a temperate tree: Evidence for environmental prediction? Austral Ecology, 37, 175-182.

Cao L, Guo C, Chen J (2017) Fluctuation in seed abundance has contrasting effects on the fate of seeds from two rapidly germinating tree species in an Asian tropical forest. Integrative Zoology, 12, 2-11.

Cao L, Wang ZY, Yan C, Chen J, Guo C, Zhang ZB (2016) Differential foraging preferences on seed size by rodents result in higher dispersal success of medium-sized seeds. Ecology, 97, 3070-3078.

Chen B, Song YC, Da LJ (2003) A review on mast seeding studies. Chinese Journal of Applied Ecology, 34, 117-120. (in Chinese with English abstract) [陈波, 宋永昌, 达良俊 (2003) 植物大年结实研究概述. 应用生态学报, 34, 117-120.]

Chen YY, Hsu SB (2011) Synchronized reproduction promotes species coexistence through reproductive facilitation. Journal of Theoretical Biology, 274, 136-144.

Crone EE, Miller E, Sala A (2009) How do plants know when other plants are flowering? Resource depletion, pollen limitation and mast-seeding in a perennial wildflower. Ecology Letters, 12, 1119-1126.

Crone EE, Rapp JM (2014) Resource depletion, pollen coupling, and the ecology of mast seeding. Annals of the New York Academy of Sciences, 1322, 21-34.

Day DE, Mintz EM, Bartness TJ (1999) Diet self-selection and food hoarding after food deprivation by Siberian hamsters. Physiology \& Behavior, 68, 187-194.

Demas GE, Bartness TJ (1999) Effects of food deprivation and metabolic fuel utilization on food hoarding by jirds (Meriones shawi). Physiology \& Behavior, 67, 243-248.

Detto M, Wright SJ, Calderon O, Muller-Landau HC (2018) Resource acquisition and reproductive strategies of tropical forest in response to the El Niño-Southern Oscillation. 
Nature Communications, 9, 913.

Du YJ, Ma KP (2012) Advancements and prospects in forest seed rain studies. Biodiversity Science, 20, 94-107. (in Chinese with English abstract) [杜彦君, 马克平 (2012) 森 林种子雨研究进展与展望. 生物多样性, 20, 94-107.]

Fletcher QE, Boutin S, Lane JE, LaMontagne JM, McAdam AG, Krebs CJ, Humphries MM (2010) The functional response of a hoarding seed predator to mast seeding. Ecology, 91, 2673-2683.

Garzon-Lopez CX, Ballesteros-Mejia L, Ordonez A, Bohlman SA, Olff H, Jansen PA (2015) Indirect interactions among tropical tree species through shared rodent seed predators: A novel mechanism of tree species coexistence. Ecology Letters, 18, 752-760.

Granados A, Bernard H, Brodie JF (2019) The influence of logging on vertebrate responses to mast fruiting. Journal of Animal Ecology, 88, 892-902.

Herrera CM, Jordano P, Guitian J, Traveset A (1998) Annual variability in seed production by woody plants and the masting concept: Reassessment of principles and relationship to pollination and seed dispersal. The American Naturalist, 152, 576-594.

Hett JM (1971) A dynamic analysis of age in sugar maple seedlings. Ecology, 52, 1071-1074.

Houle G (2001) Mast seedling in Abies balsamea, Acer saccharum and Betula alleghaniensis in an old growth, cold temperate forest of north-eastern North America. Journal of Ecology, 87, 413-422.

Jansen PA, Bongers F, Hemerik L (2004) Seed mass and mast seeding enhance dispersal by a neotropical scatter-hoarding rodent. Ecological Monographs, 74, 569-589.

Janzen DH (1971) Seed predation by animals. Annual Review of Ecology and Systematics, 2, 465-492.

Janzen DH (1976) Why bamboos wait so long to flower. Annual Review of Ecology and Systematics, 7, 347-391.

Joubert DF, Smit GN, Hoffman MT (2013) The influence of rainfall, competition and predation on seed production, germination and establishment of an encroaching Acacia in an arid Namibian savanna. Journal of Arid Environments, 91, 7-13.

Keeley JE, Bond WJ (1999) Mast flowering and semelparity in bamboos: The bamboo fire cycle hypothesis. The American Naturalist, 154, 383-391.

Kelly D (1994) The evolutionary ecology of mast seeding. Trends in Ecology \& Evolution, 9, 465-470.

Kelly D, Geldenhuis A, James A, Holland EP, Plank MJ, Brockie RE, Cowan PE, Harper GA, Lee WG, Maitland MJ, Mark AF, Mills JA, Wilson PR, Byrom AE (2013) Of mast and mean: Differential-temperature cue makes mast seeding insensitive to climate change. Ecology Letters, 16, 90-98.

Kelly D, Hart DE, Allen RB (2001) Evaluating the wind pollination benefits of mast seeding. Ecology, 82, 117-126.

Kelly D, Sork VL (2002) Mast seeding in perennial plants:
Why, how, where? Annual Review of Ecology and Systematics, 33, 427-447.

Koenig WD, Funk KA, Kraft TS, Carmen WJ, Barringer BC, Knops JMH (2012) Stabilizing selection for within-season flowering phenology confirms pollen limitation in a wind-pollinated tree. Journal of Ecology, 100, 758-763.

Koenig WD, Knops JMH (2000) Patterns of annual seed production by Northern Hemisphere trees: A global perspective. The American Naturalist, 155, 59-69.

Koenig WD, Knops JMH (2005) The mystery of masting in trees. American Scientist, 93, 340-347.

Koenig WD, Knops JMH, Carmen WJ (2010) Testing the environmental prediction hypothesis for mast-seeding in California oaks. Canadian Journal of Forest Research, 40, 2115-2122.

Koenig WD, Knops JMH, Carmen WJ, Pearse IS (2015) What drives masting? The phenological synchrony hypothesis. Ecology, 96, 184-192.

Koenig WD, Mumme RL, Carmen WJ, Stanback MT (1994) Acorn production by oaks in Central Coastal California: Variation within and among years. Ecology, 75, 99-109.

Kon H, Noda T, Terazawa K, Koyama H, Yasaka M (2005) Evolutionary advantages of mast seeding in Fagus crenata. Journal of Ecology, 93, 1148-1155.

Lalonde RG, Roitberg BD (1992) On the evolution of masting behavior in trees: Predation or weather? The American Naturalist, 139, 1293-1304.

Li HJ, Zhang ZB (2007) Effects of mast seeding and rodent abundance on seed predation and dispersal by rodents in Prunus armeniaca (Rosaceae). Forest Ecology and Management, 242, 511-517.

Monks A, Monks JM, Tanentzap AJ (2016) Resource limitation underlying multiple masting models makes mast seeding sensitive to future climate change. New Phytologist, 210, 419-430.

Montiel S, Montaña C (2000) Vertebrate frugivory and seed dispersal of a Chihuahuan Desert cactus. Plant Ecology, 146, 221-229.

Moore JE, McEuen AB, Swihart RK, Contreras TA, Steele MA (2007) Determinants of seed removal distance by scatter-hoarding rodents in deciduous forests. Ecology, 88, 2529-2540.

Moreira X, Abdala-Roberts L, Linhart YB, Mooney KA (2014) Masting promotes individual- and population-level reproduction by increasing pollination efficiency. Ecology, 95, 801-807.

Nathan R, Muller-Landau HC (2000) Spatial patterns of seed dispersal, their determinants and consequences for recruitment. Trends in Ecology \& Evolution, 15, 278-285.

Nilsson SG, Wastljung U (1987) Seed predation and cross-pollination in mast-seeding beech (Fagus sylvatica) patches. Ecology, 68, 260-265.

Norghauer JM, Newbery DM (2011) Seed fate and seedling 
dynamics after masting in two African rain forest trees. Ecological Monographs, 81, 443-468.

Norton DA, Kelly D (1988) Mast seeding over 33 years by Dacrydium cupressinum Lamb. (rimu) (Podocarpaceae) in New Zealand: The importance of economies of scale. Functional Ecology, 2, 399-408.

Nussbaumer A, Waldner P, Apuhtin V, Aytar F, Benham S, Bussotti F, Eichhorn J, Eickenscheidt N, Fabianek P, Falkenried L, Leca S, Lindgren M, Manzano Serrano MJ, Neagu S, Nevalainen S, Pajtik J, Potočić N, Rautio P, Sioen G, Stakenas V, Tasdemir C, Thomsen IM, Timmermann V, Ukonmaanaho L, Verstraeten A, Wulff S, Gessler A (2018) Impact of weather cues and resource dynamics on mast occurrence in the main forest tree species in Europe. Forest Ecology and Management, 429, 336-350.

Ostfeld RS, Canham CD, Oggenfuss K, Winchcombe RJ, Keesing F (2006) Climate, deer, rodents, and acorns as determinants of variation in lyme-disease risk. PLoS Biology, 4, e145.

Ostfeld RS, Jones CG, Wolff JO (1996) Of mice and mast: Ecological connections in eastern deciduous forests. BioScience, 46, 323-330.

Pearse IS, Koenig WD, Kelly D (2016) Mechanisms of mast seeding: Resources, weather, cues, and selection. New Phytologist, 212, 546-562.

Pearse IS, Koenig WD, Knops JMH (2014) Cues versus proximate drivers: Testing the mechanism behind masting behavior. Oikos, 123, 179-184.

Pearson AK, Pearson OP, Gomez IA (1994) Biology of the bamboo Chusquea culeou (Poaceae: Bambusoideae) in Southern Argentina. Vegetatio, 111, 93-126.

Perez-Ramos IM, Padilla-Diaz CM, Koenig WD, Maranon T (2015) Environmental drivers of mast-seeding in Mediterranean oak species: Does leaf habit matter? Journal of Ecology, 103, 691-700.

Pesendorfer MB, Koenig WD (2016) The effect of within-year variation in acorn crop size on seed harvesting by avian hoarders. Oecologia, 181, 97-106.

Pesendorfer MB, Koenig WD (2017) Competing for seed dispersal: Evidence for the role of avian seed hoarders in mediating apparent predation among oaks. Functional Ecology, 31, 622-631.

Pesendorfer MB, Koenig WD, Pearse IS, Knops JMH, Funk KA, Whitney K (2016a) Individual resource limitation combined with population-wide pollen availability drives masting in the valley oak (Quercus lobata). Journal of Ecology, 104, 637-645.

Pesendorfer MB, Sillett TS, Morrison SA, Kamil AC (2016b) Context-dependent seed dispersal by a scatter-hoarding corvid. Journal of Animal Ecology, 85, 798-805.

Piovesan G, Adams JM (2005) The evolutionary ecology of masting: Does the environmental prediction hypothesis also have a role in mesic temperate forests? Ecological Research,
20, 739-743.

Sala A, Hopping K, McIntire EJ, Delzon S, Crone EE (2012) Masting in whitebark pine (Pinus albicaulis) depletes stored nutrients. New Phytologist, 196, 189-199.

Salisbury EJ (1942) The Reproductive Capacity of Plants. G. Bell and Sons, Ltd., London.

Schauber EM, Kelly D, Turchin P, Simon C, Lee WG, Allen RB, Payton IJ, Wilson PR, Cowan PE, Brockie RE (2002) Masting by eighteen New Zealand plant species: The role of temperature as a synchronizing cue. Ecology, 83, 1214 $-1225$.

Selås V (1997) Cyclic population fluctuations of herbivores as an effect of cyclic seed cropping of plants: The mast depression hypothesis. Oikos, 80, 257-268.

Selås V (2016) Seventy-five years of masting and rodent population peaks in Norway: Why do wood mice not follow the rules? Integrative Zoology, 11, 388-402.

Silvertown JW (1980) The evolutionary ecology of mast seeding in trees. Biological Journal of the Linnean Society, 14, 235-250.

Smith CC, Hamrick JL, Kramer CL (1990) The advantage of mast years for wind pollination. The American Naturalist, 136, 154-166.

Smith HM, Samach A (2013) Constraints to obtaining consistent annual yields in perennial tree crops. I. Heavy fruit load dominates over vegetative growth. Plant Science, 207, 158-167.

Solbreck C, Knape J (2017) Seed production and predation in a changing climate: New roles for resource and seed predator feedback? Ecology, 98, 2301-2311.

Sork VL (1983) Mammalian seed dispersal of pignut hickory during three fruiting seasons. Ecology, 64, 1049-1056.

Sork VL (1993) Evolutionary ecology of mast-seeding in temperate and tropical oaks (Quercus spp.). Vegetatio, 107/108, 133-147.

Sun IF, Chen YY, Hubbell SP, Wright SJ, Noor NSM (2007) Seed predation during general flowering events of varying magnitude in a Malaysian rain forest. Journal of Ecology, 95, 818-827.

Sun MY, Ma QL, Tian SL, Wang JD, Lu JQ (2011) Effects of seed productivity on dispersal of nuts of Quercus variabilis by rodents. Acta Theriologica Sinica, 31, 265-271. (in Chinese with English abstract) [孙明洋, 马庆亮, 田澍辽, 王建东, 路纪琪 (2011) 种子产量对鼠类扩散栓皮栋坚 果的影响. 兽类学报, 31, 265-271.]

Theimer TC (2001) Seed scatterhoarding by white-tailed rats: Consequences for seedling recruitment by an Australian rain forest tree. Journal of Tropical Ecology, 17, 177-189.

Tisch PA, Kelly D (1998) Can wind pollination provide a selective benefit to mast seeding in Chionochloa macra (Poaceae) at Mt Hutt, New Zealand? New Zealand Journal of Botany, 36, 637-643.

Usinowicz J, Chang-Yang CH, Chen YY, Clark JS, Fletcher C, 
Garwood NC, Hao ZQ, Johnstone J, Lin YC, Metz MR, Masaki T, Nakashizuka T, Sun IF, Valencia R, Wang YY, Zimmerman JK, Ives AR, Wright SJ (2017) Temporal coexistence mechanisms contribute to the latitudinal gradient in forest diversity. Nature, 550, 105-108.

Vacchiano G, Ascoli D, Berzaghi F, Lucas-Borja ME, Caignard T, Collalti A, Mairota P, Palaghianu C, Reyer CPO, Sanders TGM, Schermer E, Wohlgemuth T, Hacket-Pain A (2018) Reproducing reproduction: How to simulate mast seeding in forest models. Ecological Modelling, 376, 40-53.

Vander Wall SB (1990) Food Hoarding in Animals. University of Chicago Press, Chicago.

Vander Wall SB (2002) Masting in animal-dispersed pines facilitates seed dispersal. Ecology, 83, 3508-3516.

Vander Wall SB (2010) How plants manipulate the scatter-hoarding behaviour of seed-dispersing animals. Philosophical Transactions of the Royal Society B, 365, 989-997.

Vander Wall SB, Beck MJ (2012) A comparison of frugivory and scatter-hoarding seed-dispersal syndromes. Botanical Review, 78, 10-31.

Vander Wall SB, Kuhn KM, Beck MJ (2005) Seed removal, seed predation, and secondary dispersal. Ecology, 86, 801-806.

Waller DM (1993) How does mast-fruiting get started? Trends in Ecology \& Evolution, 8, 122-123.

Wang J, Zhang B, Hou X, Chen XN, Han N, Chang G (2017) Effects of mast seeding and rodent abundance on seed predation and dispersal of Quercus aliena (Fagaceae) in Qinling Mountains, Central China. Plant Ecology, 218, 855-865.

West-Eberhardt MJ (2003) Developmental Plasticity and Evolution. Oxford University Press, New York.

Williamson GB, Ickes K (2002) Mast fruiting and ENSO cycles: Does the cue betray a cause? Oikos, 97, 459-461.

Wong R, Jones CH (1985) A comparative analysis of feeding and hoarding in hamsters and gerbils. Behavioural Processes, 11, 301-308.

Wright SJ, Carrasco C, Calderon O, Paton S (1999) The El Niño Southern Oscillation, variable fruit production, and famine in a tropical forest. Ecology, 80, 1632-1647.

Wright SJ, Muller-Landau HC, Calderón O, Hernandéz A (2005) Annual and spatial variation in seedfall and seedling recruitment in a neotropical forest. Ecology, 86, 848-860.
Xiao ZS, Gao X, Zhang ZB (2013a) Sensitivity to seed germination schedule by scatter-hoarding Pére David's rock squirrels during mast and non-mast years. Ethology, 119, 472-479.

Xiao ZS, Huang XQ (2019) How seed defense and seed abundance predict dispersal and survival patterns in Camellia. Integrative Zoology, 15, 103-114.

Xiao ZS, Zhang ZB, Krebs CJ (2013b) Long-term seed survival and dispersal dynamics in a rodent-dispersed tree: Testing the predator satiation hypothesis and the predator dispersal hypothesis. Journal of Ecology, 101, 1256-1264.

Xiao ZS, Zhang ZB, Krebs CJ (2015) Seed size and number make contrasting predictions on seed survival and dispersal dynamics: A case study from oil tea Camellia oleifera. Forest Ecology and Management, 343, 1-8.

Xiao ZS, Zhang BZ, Wang YS (2005) The effects of seed abundance on seed predation and dispersal by rodents in Castanopsis fargesii (Fagaceae). Plant Ecology, 177, 249-257.

Yang XF, Yan C, Gu HF, Zhang ZB (2020) Interspecific synchrony of seed rain shapes rodent-mediated indirect seed-seed interactions of sympatric tree species in a subtropical forest. Ecology Letters, 23, 45-54.

Yin DS, Shen HL (2016) Research review on the characteristics of mast seeding in forest trees and its influencing factors. Chinese Journal of Ecology, 35, 542-550. (in Chinese with English abstract) [殷东生, 沈海 龙 (2016) 林木大年结实特征及其影响因素研究进展. 生态学杂志, 35, 542-550.]

Zhang HM, Cheng JR, Xiao ZS, Zhang ZB (2008) Effects of seed abundance on seed scatter-hoarding of Edward's rat (Leopoldamys edwardsi Muridae) at the individual level. Oecologia, 158, 57-63.

Zwolak R, Bogdziewicz M, Wrobel A, Crone EE (2016) Advantages of masting in European beech: Timing of granivore satiation and benefits of seed caching support the predator dispersal hypothesis. Oecologia, 180, 749-758.

Żywiec M, Holeksa J, Ledwoń M (2012) Population and individual level of masting in a fleshy-fruited tree. Plant Ecology, 213, 993-1002.

(责任编委：王少鹏 责任编辑：时意专) 